P18.04 A NOVEL ANALYTIC FRAMEWORK TO INVESTIGATE VOLUNTARY MEDICAL MALE CIRCUMCISION PROGRAM EFFICIENCY GAINS THROUGH SUB-POPULATION PRIORITISATION: INSIGHTS FROM APPLICATION TO ZAMBIA

${ }^{1}$ Susanne F Awad* ${ }^{2,3}$ Sema K Sgaier, ${ }^{4}$ Bushimbwa C Tambatamba, ${ }^{1}$ Yousra A Mohamoud, ${ }^{2}$ Fiona K Lau, ${ }^{5}$ Jason B Reed, ${ }^{6}$ Emmanuel Njeuhmeli, 1,7,8 Laith J Abu-Raddad. ${ }^{1}$ Infectious Disease Epidemiology Group, Weill Cornell Medical College - Qatar, Cornell University, Qatar Foundation - Education City, Doha, Qatar; ${ }^{2}$ Integrated Delivery, Global Development Program, Bill \& Melinda Gates Foundation, Seattle, Washington, USA; ${ }^{3}$ Department of Global Health, University of Washington, Seattle, USA; ${ }^{4}$ Ministry of Community Development and Mother and Child Health, Lusaka, Zambia; ${ }^{5}$ Office of the US Global AIDS Coordinator, Washington, District of Columbia, USA; ${ }^{6}$ United States Agency for International Development, Washington, District of Columbia, USA; 'Department of Healthcare Policy and Research, Weill Cornell Medical College, Cornell University, New York, New York, USA; ${ }^{8}$ Vaccine and Infectious Disease Division, Fred Hutchinson Cancer Research Center, Seattle, Washington, USA

\subsection{6/sextrans-2015-052270.627}

Introduction Countries in sub-Saharan Africa are scaling-up voluntary male medical circumcision (VMMC) as an HIV intervention. Emerging challenges in these programs call for increased focus on program efficiency (optimising impact while minimising cost). A novel analytic approach was developed to determine how sub-population prioritisation can increase program efficiency, as applied to Zambia.

Methods A population-level mathematical model was constructed describing the HIV epidemic and impact of VMMC programs (Age-Structured Mathematical (ASM) model). The model stratified the population according to sex, circumcision status, age group, sexual-risk group, HIV status and infection stage. A three-level conceptual framework was also developed to determine the maximum epidemic impact and program efficiency through sub-population prioritisation, based on age, geography, and risk profile.

Results In the baseline scenario, achieving 80\% VMMC coverage by 2017 among males $15-49$ years, 12 VMMCs are needed per HIV infection averted (effectiveness), the cost per infection averted (cost-effectiveness) is $\$ 1,089$, and the number of infections averted is 306,000 . Through age-group prioritisation, effectiveness ranged from 11 (20-24 age-group) to 36 (45-49 age-group) circumcisions per infection averted. Cost-effectiveness ranged from $\$ 888$ (20-24 age-group) to $\$ 3,300$ (45-49 agegroup). Circumcising age groups $10-14,15-19$ or $20-24$ achieved the largest HIV incidence rate reduction. Prioritising age groups $15-24,15-29$ or $15-34$ achieved the greatest program efficiency. Through geographical prioritisation, effectiveness ranged from 9 to 12 circumcisions per infection averted. Prioritising Lusaka, the province with the highest HIV prevalence, achieved the highest effectiveness. Through risk-group prioritisation, prioritising highest risk groups achieved the highest effectiveness, with only one VMMC per infection averted, while prioritising the lowest risk group required 80 times more VMMCs.

Conclusion Epidemic impact and efficiency of VMMC programs can be improved by prioritising males in age group 15-34, geographical areas with higher HIV prevalence than the national and high sexual-risk groups.

Disclosure of interest statement This publication is based on research funded by the Bill and Melinda Gates Foundation. Infrastructure support was provided by the Biostatistics, Epidemiology, and Biomathematics Research Core at the Weill Cornell Medical College in Qatar.
The content of this manuscript is the sole responsibility of the authors. The information provided here is not official US Government information and does not necessarily represent the views or positions of United States Agency for International Development, the United States Government, or the Bill and Melinda Gates Foundation.

\section{P18.05 CONTRIBUTION OF BIOMARKERS TO THE VALIDATION OF SELF-REPORTED CONDOM USE IN A TREATMENT AS PREVENTION AND A PRE-EXPOSURE PROPHYLAXIS DEMONSTRATION STUDY AMONG FEMALE SEX WORKERS}

${ }^{1,2,3} \mathrm{M}$ Alary ${ }^{*},{ }^{4} \mathrm{~L}$ Béhanzin, ${ }^{4} \mathrm{FA}$ Guédou, ${ }^{4} \mathrm{~N}$ Geraldo, ${ }^{4} \mathrm{E}$ Goma Mastétsé, ${ }^{4} \mathrm{~N}$ Singbo, ${ }^{5} \mathrm{JC}$ Sossa, ${ }^{6,7} \mathrm{DM}$ Zannou, ${ }^{1,2} \mathrm{~K}$ Giguère. ${ }^{1}$ Centre de Recherche Du CHU de Québec, Québec, Canada; ${ }^{2}$ Département de Médecine Sociale Et Préventive, Université Laval, Québec, Canada; ${ }^{3}$ nstitut National de Santé Publique Du Québec, Québec, Canada; ${ }^{4}$ Dispensaire IST, Centre de Santé Communal de Cotonou 1, Cotonou, Bénin; ${ }^{5}$ Programme National de Lutte Contre Le Sida Et Les IST (PNLS-IST), Cotonou, Bénin; ${ }^{6}$ Faculté Des Sciences de La Santé, Université d'Abomey-Calavi, Cotonou, Bénin; ${ }^{7}$ Centre National Hospitalier Universitaire HMK de Cotonou, Bénin

\subsection{6/sextrans-2015-052270.628}

Introduction The phenomenon of condom migration remains a major concern whenever a new HIV prevention approach is being considered to strengthen the existing preventive arsenal. We are currently measuring the baseline condom use at the recruitment visit of an on-going demonstration study on preexposure prophylaxis (PrEP) and treatment as prevention (TasP), involving female sex workers (FSWs) in Cotonou, Benin, West Africa.

Methods During the recruitment visit, data on unprotected sex were collected through face-to-face interviews, from the first 112 FSWs with available biomarker results, recruited as of February 28, 2015. In addition, vaginal samples were tested for prostate-specific antigen (PSA) and Y chromosome DNA (YcDNA) PCR tests, to validate the self-reported unprotected sex. Additional biomarkers include gonorrhoea and chlamydia testing using nucleic-acid amplification tests and urine pregnancy tests. Performance parameters of the self-reported unprotected sex were calculated using combined results of all biomarkers as gold standard for recent semen exposure.

Results The prevalence of recent self-reported unprotected sex with all types of sexual partners was $31.2 \%(35 / 112)$ vs. $51.8 \%$ $(58 / 112)$ according to the combined biomarkers $(\mathrm{p}=0.003$, McNemar test). Using the combined biomarkers as gold standard, the sensitivity and specificity of self-reported unprotected sex were $34.5 \%$ and $72.2 \%$, respectively. The prevalences of gonorrhoea and chlamydia at the recruitment visit were $8.0 \%$ (9/112) and 6.2\% (7/112), respectively. Either infection was present in 16 participants $(14.3 \%)$, out of whom 10 were positive for PSA or Yc-DNA PCR. The pregnancy test was positive for two participants of whom one was positive for both PSA and Yc-DNA PCR.

Conclusion These results confirm the questionable validity of self-reported condom use among FSWs. They underscore the need of supplementing self-reported data by biomarkers to improve data reliability. These early results are part of the baseline data that will be subsequently used to look for condom migration.

Disclosure of interest statement This study is funded by the Bill and Melinda Gates Foundation, and Truvada ${ }^{\circledR}$ for pre-exposure prophylaxis is provided free of charge by Gilead Sciences, Inc. 
P18.06 EARLY ANTIRETROVIRAL THERAPY AND PRE-EXPOSURE PROPHYLAXIS FOR HIV PREVENTION AMONG FEMALE SEX WORKERS IN COTONOU, BENIN: EARLY FOLLOW-UP AND ADHERENCE DATA

${ }^{1} \mathrm{FA}$ Guédou*, ${ }^{1} \mathrm{~L}$ Béhanzin, ${ }^{1} \mathrm{~N}$ Geraldo, ${ }^{1} \mathrm{E}$ Goma Mastétsé, ${ }^{1} \mathrm{~N}$ Singbo, ${ }^{2} \mathrm{JC}$ Sossa, ${ }^{3,4}$ MD Zannou, ${ }^{5,6,7} \mathrm{M}$ Alary. NJCMDM ${ }^{1}$ Dispensaire IST, Centre de Santé Communal de Cotonou 1, Cotonou, Bénin; ${ }^{2}$ Programme National de Lutte Contre Le Sida Et Les IST (PNLSIST), Cotonou, Bénin; ${ }^{3}$ Faculté Des Sciences de La Santé, Université d'Abomey-Calavi, Cotonou, Bénin; ${ }^{4}$ Centre National Hospitalier Universitaire HMK de Cotonou, Bénin; ${ }^{5}$ Centre de Recherche Du CHU de Québec, Québec, Canada; ${ }^{6}$ Département de Médecine Sociale Et Préventive, Université Laval, Québec, Canada; 'Institut National de Santé Publique Du Québec, Québec, Canada

\section{$10.1136 /$ sextrans-2015-052270.629}

Introduction To assess the feasibility and usefulness of integrating treatment as prevention (TasP) and pre-exposure prophylaxis (PrEP) with Truvada ${ }^{\circledR}$ to the combination prevention package offered to female sex workers (FSWs) in Cotonou, we are currently carrying out a demonstration project on these two HIV preventive strategies among FSWs.

Methods We are currently recruiting $100 \mathrm{HIV-infected} \mathrm{FSWs} \mathrm{for}$ TasP and 250 HIV-negative FSWs for PrEP (one-year recruitment period, followed by an additional one year of follow-up). The actual recruitment visit is preceded by a screening visit two weeks earlier in order to determine the HIV status and assess other eligibility criteria. Through follow-up visits at day 14 and then quarterly, we closely monitor treatment adherence, using various tools including a pill count strategy. For the latter, we ask participants to bring in their bottles of medication at each visit. We report here on the first five months of the clinical phase of the study.

Results From $18^{\text {th }}$ september 2014 to $28^{\text {th }}$ February 2015, we screened 154 FSWs and out of them, 85 and 34 were recruited in the PrEP and TasP arms, respectively. Median age of the participants was 34 years and 38\% were from Benin, whereas the other were from surrounding countries. To $28^{\text {th }}$ February, the overall retention rate in the study was $100 \%$ for TasP and $85 \%$ for PrEP. Most drop-outs were due to mobility and leaving the sex trade. Adherence to PrEP was $84 \%$ according to pill count. Conclusion The retention rate in the study is encouraging so far. Adherence levels, although estimated through a simple method that could lead to overestimations, could be improved and a multi-faceted adherence education program is now implemented. The availability of Truvada ${ }^{\circledR}$ blood levels will allow an objective assessment of adherence, a highly crucial parameter for the success of eventual PrEP programs.

Disclosure of interest statement This study is funded by the Bill and Melinda Gates Foundation, and Truvada ${ }^{\circledR}$ for pre-exposure prophylaxis is provided free of charge by Gilead Sciences, Inc.

\section{P18.07 HIV POST-EXPOSURE PROPHYLAXIS IN AN AUSTRALIAN REGIONAL CENTRE: PATIENT DEMOGRAPHICS AND OUTCOMES FROM 2011-2014}

1J Patruno*, 'S Yeganeh, 1,2D Russell. 'Cairns Sexual Health Service, Cairns and Hinterland Health Service District, Queensland Health; ${ }^{2}$ James Cook University

\subsection{6/sextrans-2015-052270.630}

Introduction Post-exposure prophylaxis (PEP) is a pharmacological method of preventing seroconversion following a high-risk exposure to human immunodeficiency virus (HIV). The Cairns Sexual Health Service is the central prescribing organisation for all occupational and non-occupational PEP presentations in the Cairns greater region. A retrospective analysis of presentations from 2011 to 2014 was performed to identify patterns of PEP use, re-presentation, follow-up and patient outcomes.

Methods Pharmacy records for all patients prescribed PEP at the Cairns Sexual Health Service from 01/01/2011 to 31/12/2014 were obtained. 121 presentations were identified of which 14 were excluded for incomplete patient details. A remaining 107 presentations for 81 clients were included for assessment. Charts for all 81 patients were audited with patient demographics, exposure type, follow-up attendance and outcome data collated. An excel format of the information was generated for analysis. Results Non-occupational exposures accounted for 92\% ( $\mathrm{n}=$ 98) of presentations, whilst occupational exposures accounted for $8 \%(\mathrm{n}=9) .91 \%$ of presentations were males, $75 \%$ of which were men who have sex with men. Unprotected anal intercourse was responsible for $76 \%$ of non-occupational PEP. Re-presentations for PEP were high at 24\%. Patient follow up was low with only $51 \%$ returning within 12 weeks of PEP completion, however, $13 \%$ were travellers who may have left the region. One patient who was lost to follow-up later underwent seroconversion to HIV. Two individuals with multiple presentations went on to access pre-exposure prophylaxis (PrEP).

Conclusion High rates of re-presentation and loss to follow-up indicate areas for improved response from our service. The rates of re-presentation reveal a need for further education and counselling of patients to reduce their exposure risks. At present there is no formal follow-up system at CSHS for patients prescribed PEP. Given the number of patients lost to follow-up an active system may be considered in the future.

Disclosure of interest statement No conflicts of interest to declare.

\section{P18.08 PHARMACISTS PERCEPTIONS ENGAGING IN HIV PREVENTION ACTIVITIES WITH POPULATIONS AT-RISK FOR HIV INFECTION}

${ }^{1}$ Jennifer Kibicho*, ${ }^{2}$ Jill Owczarzak, ${ }^{3}$ Tom Dilworth, ${ }^{4}$ Andy Petroll. ${ }^{1}$ College of Nursing, University of Wisconsin-Milwaukee, Milwaukee, WI, USA; ${ }^{2}$ Bloomberg School of Public Health, John Hopkins University, Baltimore, Maryland, USA; ${ }^{3}$ Wheaton Franciscan Healthcare, Milwaukee, WI, USA; ${ }^{4}$ Center for AIDS Intervention Research, Medical College of Wisconsin, Milwaukee WI 53202

\subsection{6/sextrans-2015-052270.631}

Objective 2.1 million people worldwide, including 40,000 Americans become infected with HIV each year. As medication experts and healthcare professionals that are very accessible to patients, pharmacists are well positioned to conduct HIV prevention activities including promotion of pre-exposure prophylaxis (PrEP) and providing access to sterile syringes to people who inject drugs (PWIDs). There is a paucity of research examining pharmacists' attitudes about providing HIV prevention services to at-risk populations. We examined pharmacists' perceptions in engaging in HIV -related counselling, condom use and PrEP.

Methods We asked 225 pharmacists from 41 US states questions about their comfort-level counselling on: 1) HIV/AIDS, 2) condom use, 3) clean needles to PWIDs and 4) PrEP use; interacting with HIV-positive 5) MSMs and 6) heterosexuals; and 7) selling needles to PWIDs. We ran individual generalised linear modelling (GLM) regressions for each question, and report our findings below.

Results Of 225 participants, nearly half (41\%) worked in the top HIV counties where the vast majority of PLWH reside. 